www.jmscr.igmpublication.org

Impact Factor (SJIF): 6.379

Index Copernicus Value: 71.58

ISSN (e)-2347-176x ISSN (p) 2455-0450

crossref DOI:_https://dx.doi.org/10.18535/jmscr/v6i4.14

Journal Of Medical Science And Clinical Research

\title{
Correlation of Median Motor Nerve Parameters with TSH Values in Newly Diagnosed Hypothyroid Patients in a Tertiary Care Centre Alappuzha
}

\author{
Authors \\ Dr Sinu Sankar $\mathbf{M}^{1}$, Dr Manju Dominic ${ }^{2}$, Dr Shaji $\mathbf{C ~ V}^{3}$ \\ ${ }^{1}$ Third year PG, Physiology, Affiliated to TCMC \\ ${ }^{2}$ Asso. Professor, Physiology \\ ${ }^{3}$ Head of the Dept. Neurology
}

\begin{abstract}
Background: Thyroid hormones play a role in growth and development of neuromuscular system. Hypothyroidism causes neurological dysfunctions like peripheral \& entrapment neuropathy, cerebellar ataxia, myxedema coma etc ${ }^{l}$. Studies show that motor symptoms increases with increase TSH levels. Nerve conduction study when conducted early in the course of disease helps in medical management of neuropathies before starting surgical treatment. This study assessed median motor parameters in recently diagnosed and untreated hypothyroid patients.

Objectives: To study the correlation between TSH values and median motor nerve parameters in newly diagnosed hypothyroid patients

Material \& Methods: 270 hypothyroid males and females with age 20 to 50 years were included. Patients with neurological disorders, diabetes mellitus, those taking drugs causing neuropathy, alcoholics and smokers were excluded. Study design was descriptive All patients underwent electrophysiological studies in the department of Neuromedicine, GTDMC, Alappuzha for one year.

Study variables were distal latency, conduction velocity and amplitude of median motor nerve, TSH, fT3 \& fT4. Median motor nerve parameters were correlated with TSH values and the results analysed statistically by SPSS software.

Results: Datas were analysed with SPSS version 18 using student $t$ test and chi square test and $p$ value $<0.05$ was considered as significant. Observations were represented both graphically and numerically.

Conclusion: In this study, strong association was found between TSH values and altered motor nerve parameters especially in distal latency. It may be due to entrapment neuropathy where deposition of mucinous substances around the peripheral nerves occurs. Early diagnosis and treatment can prevent the impairment of motor conduction parameters.

Keywords: Hypothyroidism, median nerve, Nerve conduction study.
\end{abstract}

\section{Background}

Thyroid gland maintains various metabolic functions by stimulating the oxygen consumption in most of the cells of our body and is necessary for their growth and maturation. WHO estimates that about two billion people are iodine deficient, based on urine excretion data. In India iodine deficiency accounts for 27 per 1000 . According to the Indian Thyroid Society about 42 million people in India suffer from diseases related to 
thyroid gland with 4 times common in women. Thyroid hormones play a role in growth and development of neuromuscular system.

Hypothyroidism causes neurological dysfunctions like peripheral \& entrapment neuropathy, cerebellar ataxia, myxedema coma etc. ${ }^{1}$ Hypothyroidism is estimated to affect $3.8-4.6 \%$ of general population.

Subclinical hypoyhtroidism affects $3-15 \%$ of the adult population. ${ }^{1}$ The thyroid gland secretes triiodothyronine (T3) and thyroxine (T4) hormones which play an important role in tissue development and metabolism. They activate nuclear transcription of a large number of genes and in almost all the cells of the body and exert a number of effects on the neuromuscular system and brain. ${ }^{2}$ The prevalence of neuromuscular disorders related to thyroid dysfunction has been reported to be between 20-80 \%. Peripheral neuropathy usually develops insidiously over a long 2 period of time either due to irregular consumption of drugs or lack of thyroid hormone replacement. Deposition of glycosaminoglycans in nerves and soft tissues surrounding them with resultant axonal degeneration and secondary segmental demyelination forms the pathological basis of peripheral nerve dysfunction. ${ }^{4}$ Sensory and motor symptoms are usually the presenting features of neuropathy. The common complaints of motor neuropathy are muscle weakness, loss of control of coordination, muscle twitching and paralysis. $^{5}$

Nerve conduction study (NCS) is an electrodiagnostic technique to study functional status of the peripheral nerves and establish the type and degree of abnormalities of the nerves. It is now widely used not only for the precise localization of neural lesions, but also for the accurate characterization of the peripheral nerve functions. ${ }^{6}$ The most commonly performed tests are motor conduction studies, sensory conduction studies and late responses (F-waves and $\mathrm{H}$ Reflexes). ${ }^{17}$

Nerve Conduction studies can be defined as recording of a peripheral neural impulse at some location distant from the site where a propagating action potential is induced in a peripheral nerve. The technique consists of an electrical stimulation of somatic nerves and recording of the evoked potentials, either from the muscles or from the nerves themselves. ${ }^{6}$ Nerve conduction studies allow us to accurately localize focal lesions. It assess the shape, amplitude, latency, and conduction velocity of an electrical signal conducted over the tested nerve. They can help to determine whether the neuropathy is the result of damage to the axons (axonal neuropathy) or the myelin (demyelinating neuropathy), or both (mixed). ${ }^{18}$ The motor nerve conduction studies are more sensitive in detecting early or mild disorders of peripheral nerves.

The median nerve entrapment at the wrist caused by the deposition of mucinous material in the tissues surrounding the nerve is one of the most frequent causes of peripheral nerve damage in hypothyroidism. Females are about four times more susceptible to hypothyroidism compared to males. ${ }^{8}$ Entrapment neuropathy like CTS is nearly ten times more common in women than in men. Many electrophysiological studies in hypothyroid patients noted a significant relationship between female gender and entrapment neuropathies. ${ }^{10}$

Studies show that motor symptoms increases with increase TSH levels. Nerve conduction study when conducted early in the course of disease helps in medical management of neuropathies before starting surgical treatment. This study assessed median motor parameters in recently diagnosed and untreated hypothyroid patients.

\section{Objective}

To study the correlation between TSH values and median motor nerve parameters in newly diagnosed hypothyroid patients

\section{Materials and Methods}

After obtaining Institutional research committe and Ethics committe approval, newly diagnosed hypothyroid patients (TSH values above and free T3 and T4 below normal) were taken from 
Medicine OP department. All patients underwent nerve conduction studies in the Neurology department. Study variables were distal latency, amplitude, conduction velocity of median nerves. Data collected in a prestructured proforma, written consents were obtained. Patients were assured that their information will be kept confidential. Serum concentration of freeT3, T4 and TSH, RBS were measured in clinical pathological lab for all patients. All newly detected 270 hypothyroid patients underwent electrodiagnostic studies in the EMG department of neurology, Government Medical College, Alappuzha.

\section{Inclusion Criteria}

Newly diagnosed hypothyroid patients (6 months duration) with age group 20 to 50 years. Both males and females were included.

\section{Exclusion Criteria}

Patients with age less than 20 and more than 50 years of age, those with neurological disorders, diabetes mellitus, those taking drugs causing neuropathy, alcoholics and smokers were excluded

\section{Study Variables}

Distal latency, conduction velocity and amplitude of median motor nerve were noted bilaterally with the help of electrodiagnostic machine in the neuromedicine department. RBS, TSH, free T3, freeT4 values were noted

\section{Data Collection}

Prestructural proforma - detailed medical history, family history treatment history and clinical examination were done. Hormones were measured by radioimmunoassay (RIA) for $\mathrm{T} 3$ and $\mathrm{T} 4$, immunoradiometric assay (IRMA) for TSH. Nerve conduction studies were done by electrophysiological method with a standard machine in the neuromedicine department. Motor conduction parameters like distal latency, amplitude and conduction velocity were noted for median nerves in all patients

\section{Observations and Results}

The present study was a descriptive study and the data was analysed using SPSS version 18. The mean and standard deviation of each motor conduction parameters were calculated. The significance of the difference of mean of each parameter was analysed using the independent samples $t$ test. The $p$ value of $<0.01$ was taken as the level ofsignificance.

\section{Correlation between TSH values and conduction status}

Table showing correlation between motor parameters and TSH values

\begin{tabular}{|l|c|c|c|c|}
\hline \multirow{2}{*}{$\begin{array}{l}\text { Median Motor } \\
\text { parameters }\end{array}$} & \multicolumn{2}{|c|}{ Right } & \multicolumn{2}{c|}{ Left } \\
\cline { 2 - 5 } $\begin{array}{l}\text { Distal latency and } \\
\text { TSH }\end{array}$ & $0.548^{* *}$ & 0.000 & $0.611^{* *}$ & 0.000 \\
\hline $\begin{array}{l}\text { Amplitude and } \\
\text { TSH }\end{array}$ & $-0.677^{* *}$ & 0.000 & $-0.643^{* *}$ & 0.000 \\
\hline $\begin{array}{l}\text { Conduction } \\
\text { velocity and TSH }\end{array}$ & $-0.614^{* *}$ & 0.000 & $-0.607 * *$ & 0.003 \\
\hline
\end{tabular}

When TSH values increase, distal latency also increases (positive correlation). At the same time, amplitude and conduction velocity decreases (negative correlation). The $\mathrm{p}$ values are highly significant (less than0.01) 
Scatter diagram for motor distal latency with TSH values

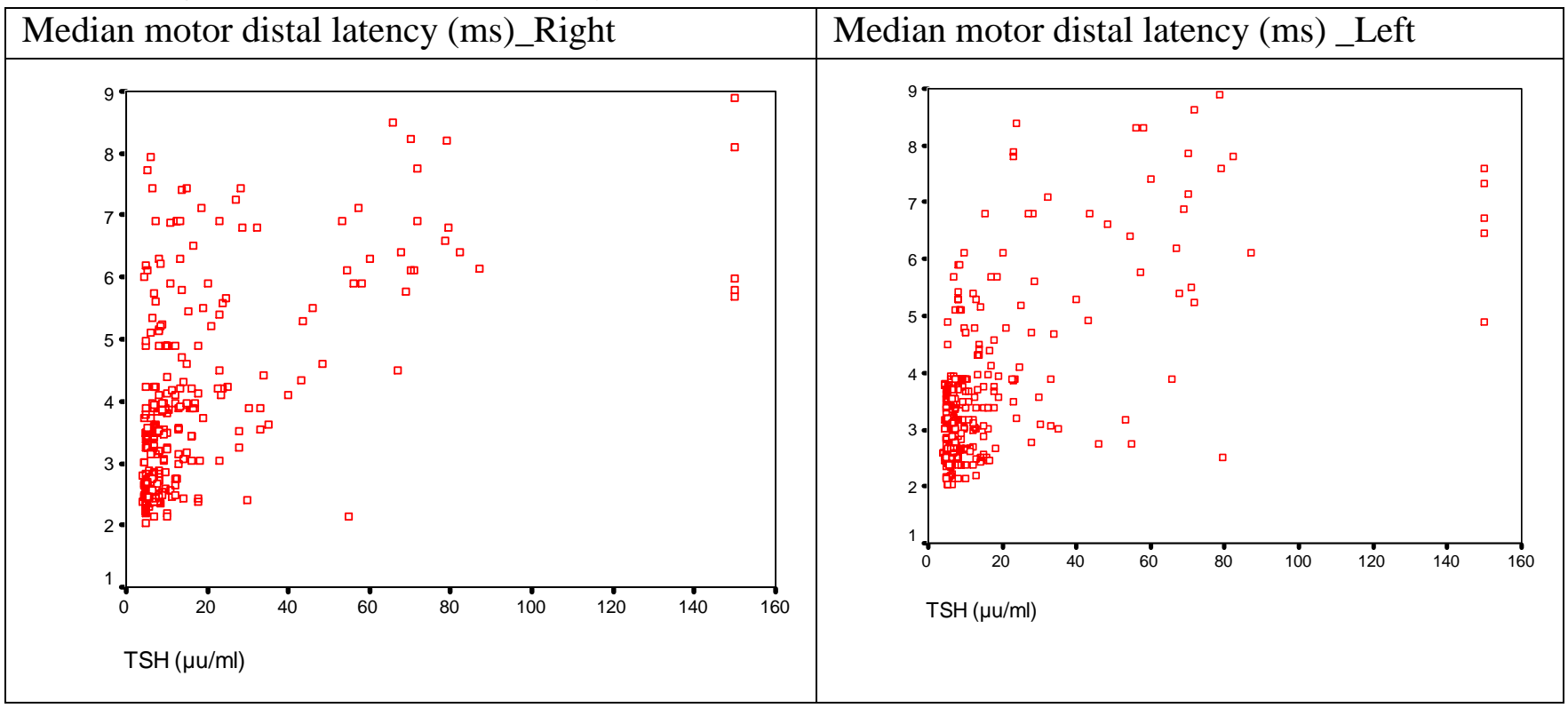

When TSH values increases distal latency increases (significant positive correlation)

Scatter diagram for motor amplitude with TSH values

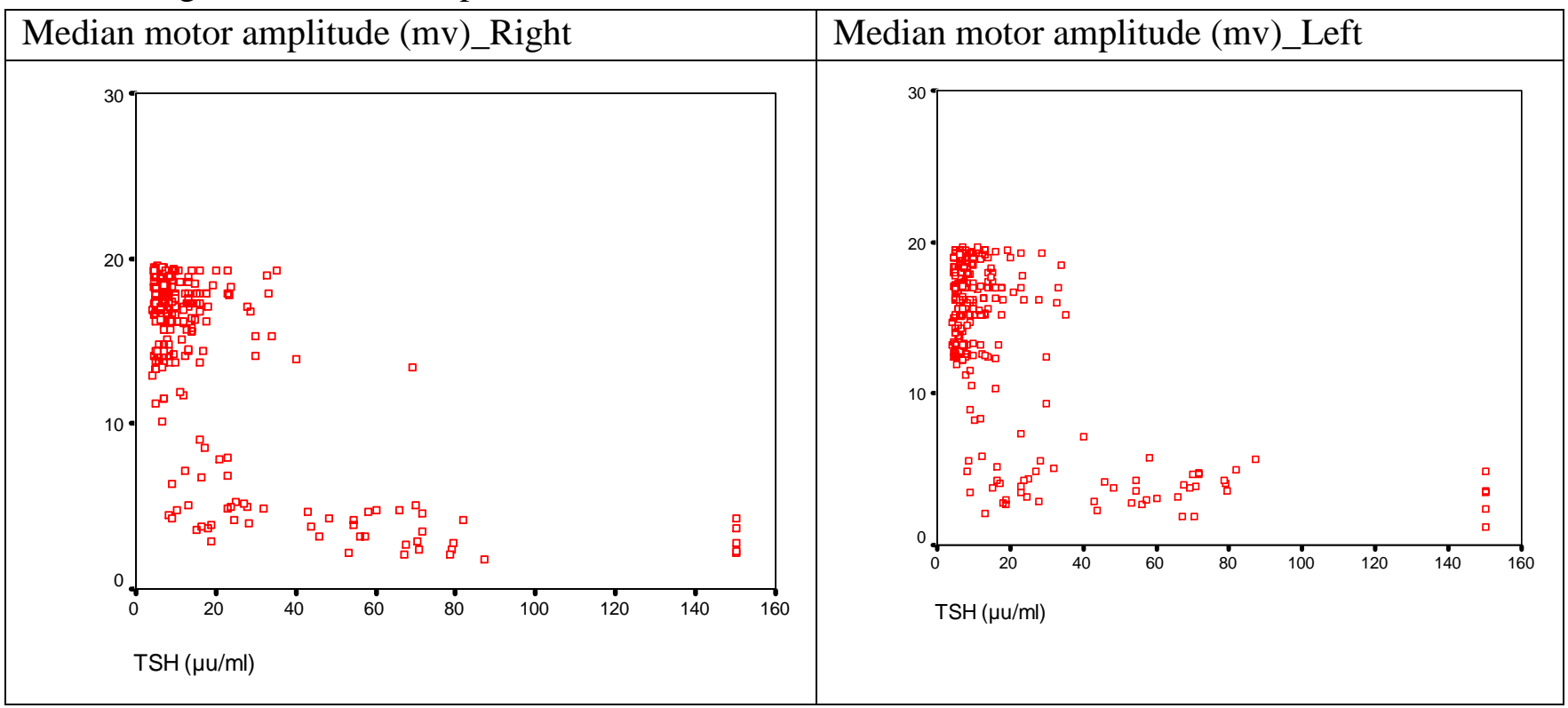

When TSH values increases amplitude decreases (significant negative correlation) 
Scatter diagram for nerve conduction velocity with TSH values

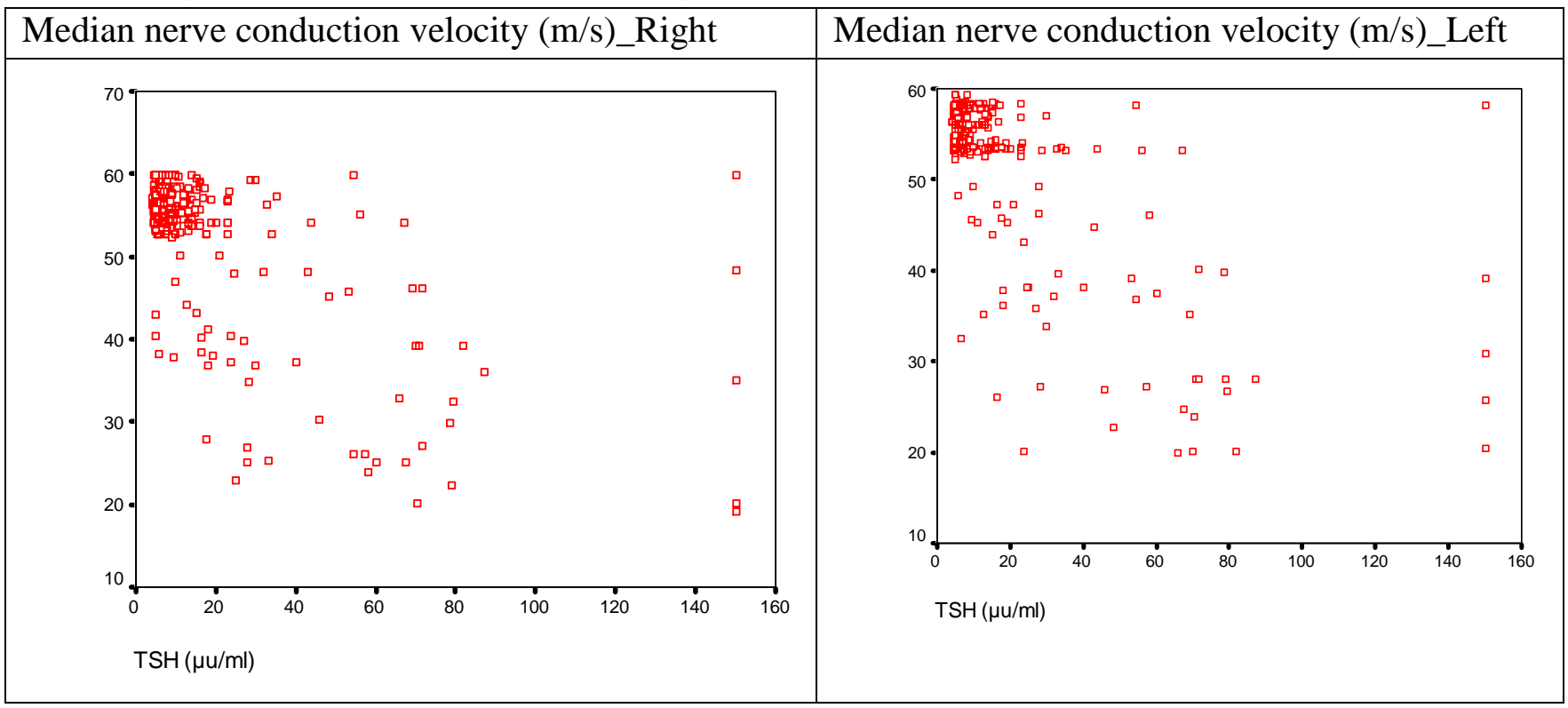

When TSH values increases conduction velocity decreases (significant negative correlation)

\section{Discussion}

Hypothyroidism is a commonest form of thyroid dysfunction which affects central and peripheral nervous system mostly. The prevalence of neuromuscular disorders related to hypothyroidism has been reported to be between $20-80 \%{ }^{6}$ Various studies have shown that hypothyroidism impairs metabolic control and increases the risk for various peripheral nerve abnormalities like entrapment and sensor motor polyneuropathy $(19,7)$. In the present study 270 newly detected hypothyroid patients were subjected to Correlation study between TSH values and nerve conduction parameters of median motor nerve and was assessed by pearson's correlation coefficient. The study showed positive correlation between TSH values and distal latency. Distal latencies of median motor nerve increased significantly with increasing values of TSH. The $p$ value is less than 0.01. Coefficient correlation of MMDL with TSH was 0.548 in right side and in left side it was 0.611 . to myelin sheath ${ }^{15}$. There is slowing of nerve conduction velocity in demyelination. Myelination is the most important factor determining conduction velocity ${ }^{12}$. Metabolic alteration caused by hormonal imbalance affects Schwann cells, inducing a segmental demyelination ${ }^{14}$. Demyelination can also occur as a result of oxidative damage to myelin sheath ${ }^{15}$. There is slowing of nerve conduction velocity in demyelination.

Sabina yeasmin et al. reported similar correlation between TSH levels and altered motor nerve parameters. They reported significantly higher $(\mathrm{P}$ $<0.01)$ distal latency in the hypothyroid groups under study ${ }^{16}$. Here the relationships of the nerve conduction parameters with the TSH level revealed positive relationships with the median motor distal latency. TSH values increases in hypothyroid patients as the thyroid hormones are decreased. Due to this primary axonal degeneration occurs and can be demonstrated under electron microscope as shrinkage of axons, disintegration of neurotubules and neurofilaments and active axonal breakdown ${ }^{7}$. Peripheral neuropathy in hypothyroidism can be due to accumulation of mucopolysaccharides, hyaluronic acid and chondroitin sulphate in the interstitial spaces which because of their hydrophilic nature, retain water and result in weight gain. This again increases the risk of peripheral neuropathy. The increase in adipose tissue causes release of free radicals that injure nervous tissue ${ }^{11}$. Considering the next parameter, motor nerve amplitude, a 
negative correlation is observed between TSH values and amplitude. When TSH increases, values of amplitude in median motor nerves decreased and the $\mathrm{p}$ value is significant $(<0.01)$. In the present study RMMA was -0.677 and LMMA -0.643. All the values are statistically significant.

In the scatter diagram for nerve conduction velocity and $\mathrm{TSH}$, a negative correlation was observed between them. The correlation coefficient of RMNCV is -0.614 and LMNCV was -0.677. All values were stastistically significant. $\mathrm{P}$ value is $<0.01$.

In the present descriptive study of motor neuropathy done in 270 newly diagnosed hypothyroid patients, there was a significant association found between TSH values and median motor nerve parameters. The age group was between 20-50 yrs. The present study included females as majority and female gender is an independent risk factor for Carpal tunnel syndrome and fluid retention. It may be partly due to hormonal factors, but in general, it is believed to be related to a propensity to and higher frequency of musculoskeletal problems among women $^{13}$.

\section{Summary and Conclusion}

The motor nerve conduction parameters of median nerve were determined in 270 newly detected hypothyroid patients by nerve conduction studies.

In the correlation studies conducted, a strong association was found between TSH values and motor median nerve conduction parameters.

Positive correlation observed between median motor distal latency and TSH values while negative correlation was observed between amplitude and motor velocity with TSH values.

Thus it can be concluded that, as the TSH values increase, median motor parameters are impaired.

The involvement of median nerve is suggestive of entrapment neuropathy in hypothyroidism. The proposed aetiology is mononeuropathy which is due to deposition of mucinous substances in and around the peripheral nerves.
In patients with clinically overt and undiagnosed hypothyroidism, peripheral nerve dysfunction may be the main manifestation with which patients can present to the OPD.

The motor nerve involvement in hypothyroid patients would have been missed in the absence of a nerve conduction study. Since the thyroid replacement therapy can effectively alleviate the motor bundle and neuromuscular junction involvement, which is due to slow basal metabolism in hypothyroidism, early treatment can prevent the impairment of motor conduction parameters and also the irreversible nerve damage which will develop late in the disease.

So this study will help to create an awareness among the hypothyroid patients about the neurological manifestations of hypothyroidism and the importance of taking early and regular thyroid replacement therapy so as to minimize the occurance of motor neuropathy in them. Nerve conduction study will also help the physicians to start medical treatment early, before switching on to surgical procedures.

I believe further studies are needed to compare the pre and post-treatment electrophysiological findings so as to find out the effectiveness of replacement therapy in hypothyroid patients.

\section{Acknowledgement}

I wish to express my sincere gratitude to Dr.Manju Dominic MD, Associate Professor, Department of physiology, Govt. Medical College, Alappuzha without whose help, constant encouragement, support and regular advice, this thesis work would not have been possible.

I also wish to express my sincere thanks to Dr. N. Geetha MD, professor and Head, Department of physiology, Govt.TD Medical College, Alappuzha for the valuable help and suggestions in carrying out this work.

I wish to express my sincere thanks to Dr. Vijayalakshmi MD, Professor and Head, Department of physiology, Govt. Medical college Trissur, and Dr. Kalyanikutty MD, Professor and Head, Department of physiology, Govt.Medical 
college Kozhikodu, for the immense help and suggestions in carrying out this work.

I wish to thank Dr.CV Shaji MD, Professor and Head of the Department of Neurology, Alappuzha for the valuable advice and support throughout the study.

I would like to thank Dr.Legha MD, Professor and Head of the Department of Medicine, Alappuzha, for all her assistance in carrying out this work.

I am thankful to Dr. Rose Davy MD Associate professor, Department of physiology, Govt. Medical College, Alappuzha for helping me to carry out this work.

I also thank all staff members of the Department of Neurology and EMG section, Govt. Medical College Alappuzha for their support and help in this study.

I would also like to thank all teaching and non teaching staff faculties, post graduate students in Dept. of Physiology, Alappuzha for their cooperation and support.

I also thank The Principal, Medical College, Alappuzha, for providing me the facilities for the thesis work.

I would like to thank my husband Dr. Harikumar A MD Anaesthesiology, our parents and kids for their encouragement and whole hearted support.

I thankfully remember all the patients and their relatives who were central for materializing this study.

Above all, I sincerely thank The Almighty God, for enabling me to complete this thesis in time.

\section{References}

1. Prabir Sinha Roy, Arunima Chaudhuri1, Suranjan Banerjee et al. A study to detect abnormalities in nerve conduction velocities in newly diagnosed hypothyroid subjects .2015;15(2):187-92.

2. Ganong. Review of Medical Physiolgy. McGraw Hills Publications. 22nd edition. $2005 ; 317$.

3. Kristin Orstavik, Ingrid Norheim, et al. Neurology. 2006; vol. 67 :786-791
4. Williams. Textbook of Endocrinology. Elsevier publications. 13th edition.2015; 3rd section.

5. Dumitru D, Amato AA. Electrodiagnostic medicine. 2nd ed. Philadelphia; 2002; 947e948, 984.

6. Ashwini A Mahadule, Pravin S Jadhao and Mrunal S Phatak. Motor conduction parameters in recently diagnosed and untreated hypothyroidism. Ann Neurosci. 2015;22(1):6-1

7. Nemni R, Bottacchi E, Fazio R, Mamoli A, Corbo M, Camerlingo $\mathrm{M}$, et al. Polyneuropathy in hypothyroidism: Clinical, electrophysiological and morphological findings in four cases. J Neurol Neurosurg Psychiatry 1987; 50:1454-60.

8. Jameson and Weetman AP. Disorders of thyroid gland. In: Principals of Harrison's Internal Medicine.NewYork;2008.p.2230.

9. De Krom MC, De Krom CJ SF. Carpal tunnel syndrome: diagnosis, treatment, prevention and its relevance to dentistry. Ned Tijdschr Tandheelkd.2009;97-101.

10. Fariba Eslamian AB, Aghhamohammadzadeh N,Niafar et al. Electrophysiological changes in patients with untreated primary Hypothyroidism.J Clin Neurophysiol.2011;28(3):322-8

11. Adikesavan Balaraman, Goudhaman Natarajan et al. A study of nerve conduction velocity in newly diagnosed Hypothyroid patients.2013;9(4):198-201.

12. Ahmed faraz, Taha Nafeez et al. Autoimmune Hyppothyroidism in patients with Neurofibromatosis 1.2015;27(4):1-2.

13. Praveenkumar srikanteswara, Janardhan D et al. The Relationship between Nerve Conduction Study and clinical grading of carpal tunnel syndrome.2016;10(7):OC13OC18

14. Gaurav Kakkad, Nikita Bhatt, Jithendra Lakhani and SP. Electromyographic evaluation of blink reflex as a tool for 
early diagnosis of neurological dysfunction in patients of Hypothyroidism. Ann Neurosci.2013;20(3):95-8. 89

15. Gabriel Bucurescu, MD M. Neurological manifestations of thyroid disease Clinical Presentation: History, Physical, Causes 2014

16. Bagchi N, Brown TR, Parish RF Thyroid dysfunction in adults over age 55 years. A study in an urban U.S. community.1990; 150: 785-798.

17. Meier C, Bischoff A. Polyneuropathy in hypothyroidism. Clinical and nerve biopsy study of 4 cases. J Neurol 1977;215:10314.

18. MD Hend Azhary,MD Muhammed U Farooq, MD Minal Bhanushali, et al. peripheral neuropathy: Differential Diagnosis and Management. Am Fam physician.2010;81(7):887-92. 19. Ettorre B, Mariad, Graziella BV et al. Hypothyroidism and polyneuropathy.J Neurol Neurosurg Psychiat.1989;52:14203. 